Alma Mater Studiorum - Università di Bologna DEPARTMENT OF ECONOMICS

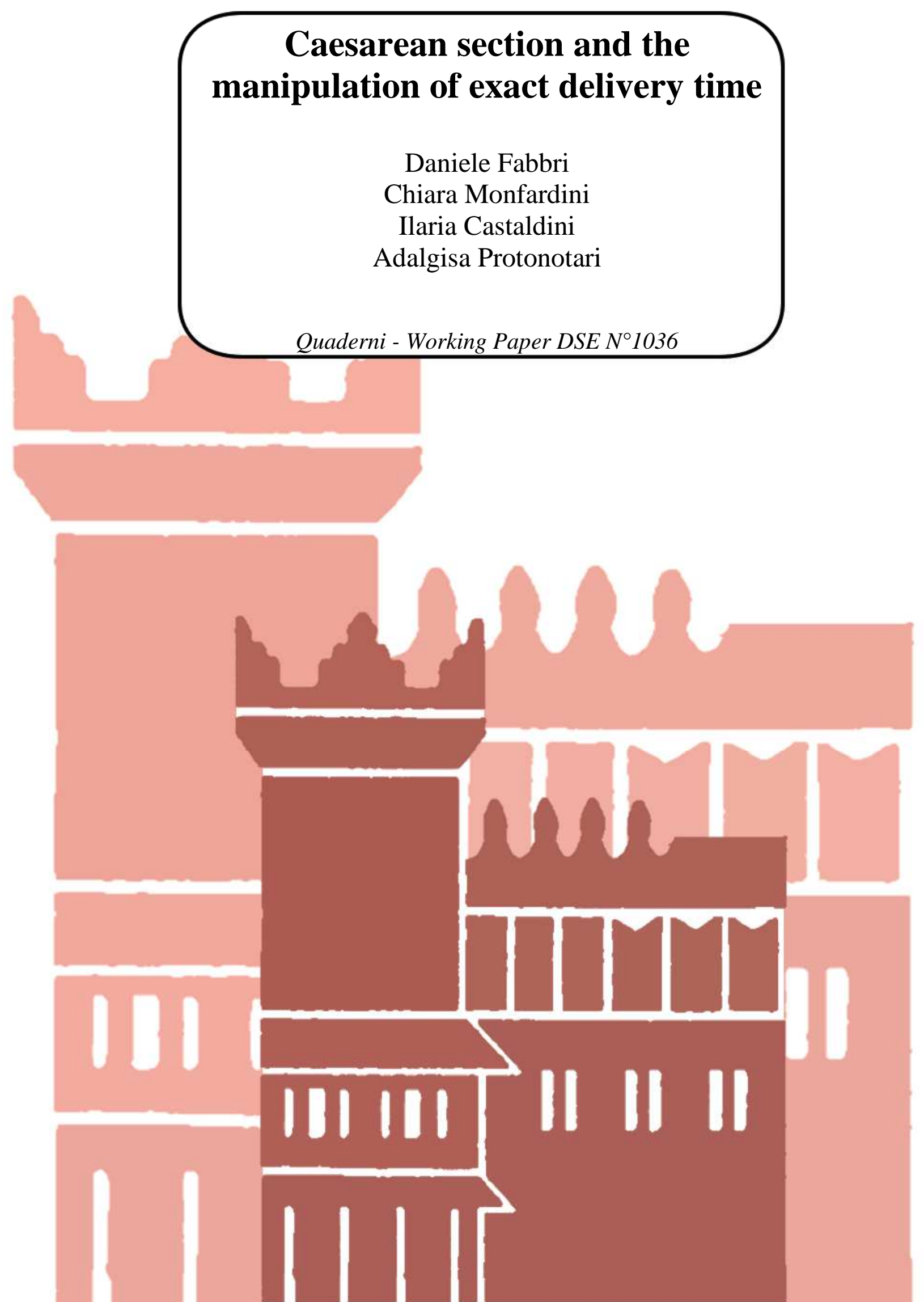




\title{
Caesarean section and the manipulation of exact delivery time
}

\author{
Daniele Fabbri \\ Department of Economics - University of \\ Bologna \\ Ilaria Castaldini \\ Planning and Control - Bologna Local \\ Health Authority
}

\author{
Chiara Monfardini \\ Department of Economics - University of \\ Bologna \\ Adalgisa Protonotari \\ Planning and Control - Bologna Local \\ Health Authority
}

October 2015

\begin{abstract}
:
Physicians are often alleged responsible for the manipulation of delivery timing. We investigate this issue in a setting that negates the influence of financial incentives behind "physician's demand induction" but allows for "risk aversion" to medical errors and "demand for leisure" motivations. Working on a sample of women admitted at the onset of labor in a big public hospital in Italy we estimate a model for the exact time of delivery as driven by individual indication to receive Caesarean Section (CS) and covariates. We find that ICS does not affect the day of delivery but leads to a circadian rhythm in the likelihood of delivery. The pattern is consistent with the postponement of high ICS deliveries in the late night $\backslash$ early morning shift. Our evidence hardly supports the manipulation of timing of births as driven by medical staff's "demand for leisure". An explanation based on "risk aversion" attitude seems more appropriate.
\end{abstract}

\section{Keywords:}

timing of delivery, physician incentives, caesarean section, scheduling

JEL codes:

I11, L23, C35, C51

\section{Acknowledgments:}

We are grateful to Randall Ellis and Yongsay Jong for helpful comments. Usual disclaimers apply.

\section{Corresponding Author:}

Daniele Fabbri, Department of Economics, University of Bologna, Piazza Scaravilli 2-40126 BOLOGNA - ITALY. Voice: +39+051+2098669. E-mail: d.fabbri@unibo.it 


\section{InTRODUCTION}

Technology for births allows for some medical manipulation of the exact timing of delivery mainly through the adoption of cesarean section (CS) and inducement of labor (Gans and Leigh (2008)). Obstetricians and patients may have reasons to anticipate or delay the exact timing of deliveries thus altering the nature's almost uniform distribution of births (Bernis and Varea (2012)). Tax benefits and inauspicious birthdates avoidance have been considered in the literature as possible motivations for patients' demand for medical manipulation of the exact day of delivery. Dickert-Conlin and Chandra (1999) and Gans and Leigh (2009) provide supportive evidence on the first mechanism; Lo (2003), Hsu et al. (2007) and Gans and Leigh (2012) find evidence of births move from inauspicious days. Since the perfect planning of a child's date of birth is unlikely without medical intervention, this evidence suggests that physicians might, to some extent, accommodate patients' demand for birthdate.

Physicians may also have their own convenience to manipulate the timing of births. Several papers provided evidence on obstetricians' preference for moving deliveries away from holidays. A drop of births on Sundays have been observed in England, USA, Canada, Australia, Germany; while Cohen (1983), studying Israel, found fewer births on Saturday and more births on Sundays. The number of births declines by 1 to 4 percent also during the days on which annual obstetricians' conferences are held (Gans et al. (2007)). Evidence on physicians preference for exact timing of birth is more limited. Brown (1996) and Spetz et al. (2001), exploring the variation of CS deliveries across time of the day, find that the probability of unplanned CS is higher and increasing in the evening (from 6 pm to midnight).

The economic literature offers three main explanations for these regularities on physicians' behavior. Whenever providers are residual claimant on net incomes physicians may tend to overuse CS vis-à-vis vaginal delivery, since the first is more generously reimbursed and generates higher net income. Consistently with the "physicians' induced demand" motivation, as treatment costs are higher in off-peak, leisure periods and weekends, CS deliveries may be less frequent then. Another explanation refers to physicians manipulating the exact time of deliveries as a defensive 
strategy to curb malpractice risks or minimize medical errors. Provided that external risks change in time, style of practice might adapt accordingly to reduce exposure as the produce of physicians' "risk aversion". A third mechanism, the so-called "demand for leisure" motivation, relates to vaginal deliveries being more time consuming than CS. As the physicians' opportunity cost of time changes in time, style of practice might change as well.

In this paper we aim to assess whether and why physicians manipulate the exact timing of delivery in the short term, i.e. focusing on urgent deliveries that are not scheduled in advance. Our case study is cast in a setting that negates the influence of financial incentives while it allows for the remaining mechanisms. We examine whether the timing of delivery is affected by women' indication for CS (ICS), as assessed at the admission, adopting a simple two-stages econometric strategy to measure individual ICS and its impact on the timing of delivery.

In the first stage, the CS probability is predicted at the individual level, based on a large set of observed clinical conditions and medical risk factors. In the second stage, we insert the predicted ICS among the regressors of a multinomial model explaining the daily shift of delivery. In the absence of any physicians' manipulation of the exact timing of deliveries to alter the nature's almost uniform distribution of births we expect delivery time to be uncorrelated with individual ICS. Therefore we interpret the finding of a significant coefficient on the ICS variable in the second stage as a signal of the existence of manipulation of the delivery time. Our identification is aided by plausible exclusion restrictions assuming that some pre-determined risk factors affect the timing of delivery only through their impact on individual indication for CS.

We find that ICS does not affect the exact day of delivery. On the contrary, we measure significant impacts within day: the higher is ICS the higher is the probability to deliver in the afternoon or evening shift ( $2 \mathrm{pm}$ to $8 \mathrm{pm}$ ), while the lower is to deliver by late night and early morning ( 2 am to $8 \mathrm{am}$ ). The same pattern is observed irrespective of admission occurring in a weekday or a weekend when the leisure motivation should be higher. Our evidence therefore implies the existence of a circadian rhythm of peaks and troughs in the likelihood of delivery for high ICS patients. We finally argue this rhythm to be produced by postponement of high ICS deliveries at night/early morning followed 
by a peak in the following afternoon shift. Thus our evidence hardly supports the manipulation of exact timing of birth to be driven by medical staff's "demand for leisure", while it favors the "risk aversion" motivation.

The paper proceeds as follow. Section 2 reviews the literature. In Section 3 we present the data, provide some background on our case study and discuss the selection of observations. Section 4 details our conceptual and empirical setup. In Section 5 we present and discuss our evidence. Section 6 contains our conclusions.

\section{BACKGROUND AND LITERATURE REVIEW}

Manipulation of the timing of birth can be maximum when delivery is scheduled in advance. This is the case for planned CS deliveries. For women having a planned CS the decision is taken that vaginal delivery, the alternative technology for birth, is least optimal due to some maternal, fetal or some other concomitant indications. ${ }^{1}$ Exact scheduling may also follow from the clinical decision to plan an induction of labor. This can be appropriate with respect to expectant management following for example a previous lower segment cesarean section (Dodd et al. (2014)), child macrosomia, prior stillbirth, post-term pregnancy, or severe preeclampsia (Nassar et al. (1998)). Planned CS and planned inducement of labor are increasingly adopted as elective modes of delivery without a medical indication (Lydon-Rochelle et al. (2007)). The manipulation of the timing of birth for clinically indicated scheduled procedure can be welfare improving as scheduling deliveries can be performed at convenient time for hospitals and physicians when resources are optimal and risks for the mother and the newborn are minimized, e.g. daytime and weekdays rather than night or weekends. For instance quite some evidence in the medical literature suggests that delivery at night is a risk factor for perinatal mortality and other adverse medical outcomes in the mother and

1 Maternal indications for cesarean section delivery include repeat cesarean delivery, pelvic abnormalities precluding engagement, obstructive lesions in the lower genital tract, genital herpes, placenta previa, HIV positivity. Fetal indications include situations in which neonatal adverse outcomes, morbidity and mortality could be decreased by the prevention of trauma and malpresentations (like preterm breech presentations), some congenital malformations or skeletal disorders, infections. 
the newborn (see for example de Graaf et al. (2010); Gould et al. (2005); Luo and Karlberg (2001)).

Recently Lefevre (2014) argued that by discarding scheduled CS, one may fail to identify the induced demand that is planned in advance by the physician. Evidence on CS deliveries being less likely on leisure days may implicitly refer to this possible effect. However her findings seems to negate this possibility. Exploiting across US States variation in the definition of public holidays on Mondays Lefevre (2014) can measure the impact of longer weekends on the probability of having a CS in periods centered around Mondays. Her findings suggests that longer weekends do not lead to any substantial increase in CS over periods centered on it as induced by physicians' demand for leisure.

A bunch of papers examine samples of deliveries comprising both planned and unplanned deliveries. Burns et al. (1995) working on deliveries in Arizona find that the probability of performing a CS increases with delivery on a Friday, and delivery between 6 am and 6 pm. In Brazil, Gomes et al. (1999) show that the likelihood of CS is higher between 7 pm and midnight and lower on Sundays. Working on data from three Greek hospitals, Mossialos et al. (2005) find that CS are more likely to occur between 8 am and 4 pm and less likely to occur on Sundays. Brown (1996) by focusing on military doctors that have no financial motivation to opt for a CS and are less subject to lawsuits than other physicians, can claim that the only non-medical incentive for CS is related to "physicians' demand for leisure". Brown (1996) finds that CS are less likely on weekends than on weekdays and more probable at the end of the day (from 6 pm to midnight). This evidence is therefore consistent with the move of CS deliveries, irrespective of being planned or not, out from weekends. However, the pattern of exact timing of deliveries is unclean and hard to interpret as the timing of planned deliveries can be driven by different motivations and different agents with respect to the timing of the urgent ones. Differently from the planned delivery case, decisions regarding unplanned deliveries, by definition, are made under pressure in the labor room, not in advance. Thus, the prevalent decision maker is clearly the physician.

A few studies take this perspective and provide evidence on unplanned deliveries. Brown (1996) finds that the probability of unplanned CS (as opposed to other mode of 
delivery including planned CS) is still higher from $6 \mathrm{pm}$ to midnight but the effect is less precisely estimated. He finds a large significant increase of unplanned CS deliveries also in the $4 \mathrm{pm}$ to $6 \mathrm{pm}$ shift that did not appear for CS in general. Using birth certificates and hospital data in California, Spetz et al. (2001) also show an intensification in unscheduled CS between $4 \mathrm{pm}$ and midnight, except for group-model HMO (Kaiser Foundation Health Plan) hospitals. Unscheduled CS are those indicated because of fetal distress or dystocia. This evidence is interpreted as suggesting that CS performed for physician convenience occur more likely in the evening hours. Group-model HMOs appear to be better able in guiding physician practice, and providing staff support to physicians so to lessen leisure-based incentive to perform CS. Spetz et al. (2001) also show that group-model HMO patients are less likely to be diagnosed with fetal distress or prolonged/dysfunctional labor implicitly suggesting that diagnosis and mode of delivery might be jointly affected by incentives provided inside the HMO model.

Similarly to the above mentioned paper, we focus here on unplanned deliveries, arguing that they represent the appropriate case study for investigating the possible existence of physicians' manipulation of the exact delivery time. Provided that the arrival rate of difficult deliveries is close to uniform in time, after controlling for medical factors, we should observe uniform rate of unplanned CS across time shifts. Hence, caseload deviations from time uniformity of CS rates could be fully attributed to some form of medical manipulation in the labor room.

As illustrated in the Introduction, we test for the existence of short run manipulation adopting a two-stage econometric approach. The individual CS probability is predicted in a first stage, resorting to additional pre-determined excluded variables, and inserted among the regressors of the second stage multinomial model that explains the daily shift of delivery. This two-step procedure accounts for the joint determination of mode and time of delivery. Simultaneity is most likely to occur due to factors (characterizing either the woman, the physician or both) unobserved to the researcher that influence both the mode of delivery and its timing. All the above-mentioned studies ignore this issue and estimate the effect of delivery time within simple models of birth mode (mainly CS vs. vaginal delivery). As a consequence, their conclusions about the time of delivery patterns are likely to be plagued by the neglected endogeneity of the time indicators. On the contrary, the predicted ICS we obtain in the first stage is an 
exogenous measure of clinical indication for urgent CS. This makes our second stage inference on its effect on the delivery shift valid and allows us to provide novel evidence on the short run manipulation of the timing of deliveries.

\section{DATA}

We examine a sample of births in Italy as reported in the Birth Certificates Database (Certificati di Assistenza al Parto - CedAP) for region Emilia-Romagna. The database collects, from Local Health Authorities (LHAs), hospital trusts and private clinics, detailed records for every delivery occurring in the region. This dataset contains information about predetermined risk factors associated with CS delivery, such as maternal age, previous cesarean sections, obstetric complications during pregnancy and mothers' general health status. Moreover, it collects many socioeconomic traits of the mother such as the level of education, the marital status, and the occupational status. We had access to the full dataset for years from 2007 to 2011.

The CEDAP dataset does not contain information on co-morbidities and complications arising at the hospital admission and during labor. Therefore, we matched CedAP data with hospital discharge records (SDO, Scheda di Dimissione Ospedaliera) for every admission to hospital associated to a delivery. SDO allowed us to recover maternal and fetal co-morbities assessed at admission, as well as a full record of problems arising during labor and delivery, classified according to ICD-9-CM. We merged the two data sources based on the patient ID.

We had access to SDO data only from one big public hospital in Emilia-Romagna, Ospedale Maggiore. Therefore, we restrict our analysis on the full caseload of deliveries occurring in this hospital between 2007 and 2011. Ospedale Maggiore ${ }^{2}$ is the third birth center in Emilia-Romagna with an average yearly caseload of about 3000 deliveries. According to the Diagnostic-Therapeutic protocol for births adopted here a one-to-one standard is in place (one midwife for every woman). The number of doctors and

2 Ospedale Maggiore is a multi-specialties center with 630 beds, 35 of which are available in the Obstetrics department. Ospedale Maggiore is equipped with a neonatology department, a neonatal intensive care unit and it is home to a first-aid obstetrics and gynecology department. 
midwives on shift is constant across days of the week, including holidays, but for neonatologists. Only one of them is on shift at night and on weekends (from Saturday afternoon to Sunday). Obstetricians are at work according to the following pattern of rotating shifts: from 7 am to $1 \mathrm{pm}$ (morning shift), from $1 \mathrm{pm}$ to $8 \mathrm{pm}$ (afternoon shift), and from $8 \mathrm{pm}$ to $7 \mathrm{am}$ (night shift). Medical staff rotates on two schedules: 8 am to 8 pm, and 8 pm to $8 \mathrm{am}$; or 8 am to $2 \mathrm{pm}, 2 \mathrm{pm}$ to $8 \mathrm{am}$, and $8 \mathrm{pm}$ to $8 \mathrm{am}$.

The total number of deliveries occurring in this hospital in the five years considered was 15.086. After excluding observations with miscoded or missing date of admission or delivery, admissions occurring in a holyday, deliveries up to more than one week after admission, and multiple deliveries, we reached a dataset comprising 13.197 valid and complete observations.

As motivated in the previous Section, given our focus on the hourly pattern of delivery treatment choice, we excluded those deliveries that were scheduled and decided in advance with respect to the day of admission. The richness of information contained in CedAP allowed us to drop from the estimation sample those deliveries explicitly classified as "elective" CS. These are scheduled in specific weekdays, leading to a pattern of non-random admission of women during the week. We excluded also those deliveries occurring upon induced labor not for emergencies arising at term. Inducement can be scheduled with some advance as complications arise in the mother or in the fetus. In this respect we kept in our estimation sample those cases being induced for labor due to premature rupture of the membranes. These selection criteria make us reasonably confident to claim that our estimation sample includes just urgent deliveries and those cases being admitted at the onset of labor. Our estimation sample contains 9,219 observations (68\% of all valid observations).

Following Brown (1996) we consider henceforth 8 possible three-hours intervals ${ }^{3}$ for delivery (see Table 1 for their definition). The spacing of these time intervals allow us to capture relevant medical staff shifts, namely the morning shift (starting at 8 am and ending at $2 \mathrm{pm}$ ), the afternoon shift (from $2 \mathrm{pm}$ to $8 \mathrm{pm}$ ) and the night shift (beginning at 8 pm until the onset of next day morning shift).

3 Brown (1996) considers either three and two hours interval. 
Figure 1 depicts the distribution of all deliveries across the day in the estimation sample and among the excluded observations. These last are heavily concentrated in the morning shift while deliveries for the women admitted for urgencies and those at the onset of labor are quite evenly distributed along the day. Further details are reported in

Table 1. In the set of excluded observations we observe, as expected, higher CS rates than for women admitted upon urgencies and at the onset of labor (57\% vs. 12\%). Among the former, CS deliveries are more frequent in the morning (77\%) and in particular in the late morning shift (86\%, 50\% higher than average), when elective CS are more frequently scheduled, declining to less than $25 \%$ in the late night shift $(57 \%$ less than average). This descriptive evidence suggests that a circadian pattern in surgeons' utilization is in place here as driven by planned CS. These are mostly scheduled in large advance during the morning shifts and drastically reduced in the rest of the day. Evidence in the literature shows that the within day concentration of other elective surgical procedures typically produced in a department of obstetrics and gynecology follow a similar circadian pattern.

On the contrary, women admitted for urgencies and at the onset of labor exhibit a more uniform distribution of CS across shifts. We notice that a peak is reached in the late afternoon shift when CS rate is $29 \%$ higher than average (see also Figure 2) while a decline occurs (especially early) in the morning. At the same time we observe that the probability to deliver is 3 to $6 \%$ higher in the morning and afternoon shift being $13 \%$ lower in the evening.

\section{Figure 1, Table 1, Figure 2 HERE}

\section{OUR THEORETICAL FRAMEWORK}

We consider a clinical set up where the woman in childbirth looks for an urgent hospital admission at term. At the hospital, the mother and fetal conditions and patient's stage of labor are evaluated by clinicians and an admission decision is taken. Upon admission the patient clinical need for a CS is assessed, based on pre-existing conditions plus on going ones. We posit that clinical assessment is made by a different agent from the one that provides the treatment. Call the first agent 1 and the second agent 2. Agent 1 elects the most appropriate type of treatment, according to a probability distribution, while 
agent 2 decides when to treat the patient. Agent 1 is a perfect agent for the patient, i.e. she develops clinical assessments considering only the wellbeing of the patient. Agent 2 can be an imperfect agent for the patient. He might act on behalf of patient's interest, thus deciding on the timing of delivery to minimize possible risks arising in the woman and the newborn. But he might anyway accommodate personal convenience when it comes to take this decision. Therefore a deliberate decision to anticipate or postpone delivery for women at the onset of labor with respect to the progression of labor might result either because of physicians' incentives to induce demand or because of physicians'risk aversion, as they would prefer to go for difficult deliveries when the largest capacity in hospital staff is on-shift or the medical staff on-shift is fresher. Given the short time span implied we call this behavior as "short-run manipulation of the exact timing of births". We exclude any strategic interaction between the two agents.

Our aim is to ascertain to what extent, if any, individuals' clinical assessment of "indication to CS" (ICS) affects the timing of delivery. We define $I C S_{i}$ as the probability, assessed by clinicians at admission, that CS is the appropriate treatment for individual $i$. In the absence of any physicians' manipulation of the exact timing of deliveries that alters the almost uniform nature's distribution of births, we expect delivery time to be uncorrelated with individual ICS and the probability of delivering in a given shift should not be affected by ICS net of other factors. On the contrary, we might argue in favor of some within day manipulation if the probability of delivering in a given shift if this is significantly affected by ICS. ${ }^{4}$

Let delivery occur in $J$ possible (8 in our model henceforth), mutually exclusive, time shifts, spanning the 24 hours in a day. Delivery by woman $i$ in shift $j$ is driven by the following latent variable:

$$
\operatorname{SHIFT}_{i j}^{*}=\gamma_{j}{ }^{\prime} x_{i}+\delta_{j} I C S_{i}+\varepsilon_{i j}
$$

4 Individual ICS is indeed continuously updated in time as labor progresses possibly reaching extreme values of zero or one. This possibility would be properly accounted for within a Dynamic Discrete Choice Model. We don't pursue this way as data on exact admission time are not available, preventing us to condition on it in a continuous time framework. We therefore condition on ICS as assessed at admission, which is a static, time invariant factor. 
such that:

$$
\begin{cases}\text { "woman i delivers in shift } j "\left(S H I F T_{i j}=1\right) & \text { if } \operatorname{SHIFT}_{i j}^{*}>0 \\ \text { "woman i delivers off shift } j^{\prime \prime}\left(\operatorname{SHIFT}_{i j}=0\right) & \text { if } \operatorname{SHIFT}_{i j}^{*} \leq 0\end{cases}
$$

where $x_{i}$ is a vector of individual's covariates, $I C S_{i}$ is the individual's assessment of clinical indication for a CS, $\varepsilon_{i j}$ are the error terms, iid standard type-1 EV. Our coefficients of interest are here the $\delta_{j} s$. As some of the $\delta_{j} s$ are different from zero we might conclude for the presence of some "within day" variation in the probability of delivery as driven by individual's ICS net of any other covariates.

A practical problem with equation 1 is that ICS is hardly observable. To address this issue we consider a simple model, in a similar vein as Chandra et al. (2012), based on patient preference and clinician assessment of the optimal treatment for the patient. In our setting at the time of admission the decision is made between two treatments for delivery: natural delivery (denoted as 0 ) and caesarean section (denoted as 1). Each treatment produces benefits to the patient of $B_{k}\left(\sigma_{i}\right)$, where $\sigma$ is the patient severity or multifactorial risk indicator. We maintain that these benefit functions are homogenous in time, i.e. individuals with the same risk profile are assumed to receive the same benefit from each delivery mode irrespective of the exact admission time.

The utility to the patient from each treatment is as follows:

$$
\begin{aligned}
& U_{i}(0)=B_{0}\left(\sigma_{i}\right)+\theta_{i 0} \\
& U_{i}(1)=B_{1}\left(\sigma_{i}\right)+\theta_{i 1}
\end{aligned}
$$

where $i$ denotes individuals. $\theta_{i 0}$ and $\theta_{i 1}$ are woman-specific error terms that capture heterogeneity in the benefits of each treatment to that patient (as for example the part due to preferences about side-effects), independent from $\sigma_{i}$.

We assume that clinicians are perfect agents for their patients knowing everything about benefits but for person-specific preference terms. Their distribution over the admitted caseload is known to the clinician, who will choose treatment 1 over treatment 0 provided $U_{i}(1)>U_{i}(0)$. In particular, a caesarean section delivery will be advised for patient $i$ if:

$$
\theta_{i 1}-\theta_{i 0}>B_{0}\left(\sigma_{i}\right)-B_{1}\left(\sigma_{i}\right)
$$


Normalizing with respect to the utility of alternative 0 we reach the following definition for ICS of individual $i$ :

$$
\begin{aligned}
& I C S_{i}=\operatorname{Pr}(C S=1) \\
& =\operatorname{Prob}\left(N B\left(\sigma_{i}\right)+u_{i}>0\right) \\
& =\operatorname{Prob}\left(u_{i}<N B\left(\sigma_{i}\right)\right) \\
& =F\left(N B\left(\sigma_{i}\right)\right)
\end{aligned}
$$

where $N B\left(\sigma_{i}\right)$ is the observable net benefit, as assessed by the clinician at admission, of pursuing CS with respect to natural delivery for individual with risk profile $\sigma_{i}$, while $u_{i}$ is the unobservable, patient specific, net benefit of CS with respect to natural delivery.

To operationalize the above measure for ICS we assume a i.i.d. standard normal distribution for $u_{i}$ and a linear benefit function, and thus estimate a probit model for the dichotomous variable $C S_{i}$ such that individual $i$ :

$$
\left\{\begin{array}{l}
\text { "Deliver with a caesarean section" } C S_{i}=1 \text { if } N B\left(\sigma_{i}\right)+u_{i}>0 \\
\text { "Deliver with a natural delivery" } C S_{i}=0 \text { if } N B\left(\sigma_{i}\right)+u_{i} \leq 0
\end{array}\right.
$$

Upon maximum likelihood estimation we obtain individuals' predicted probabilities of CS. These predicted probabilities are based on the entire set of therapeutic choices observed over the sample time span and thus reflect the best classification of patients' ICS according to the style of practice adopted at the hospital. We then plug these estimates into the multinomial model [1] for daily shift of delivery to test whether the probability of delivery exhibit some "within day" variation driven by individual's ICS net of any other covariates. Its impact reflects, for women admitted at the onset of labor, the possibility for "short-run manipulation of the exact timing of deliveries" decided by agent 2 in our framework. Inference about the parameters in the second stage model must take into account the presence of a generated regressor. To this purpose, we resort to bootstrapped standard errors.

This two stage approach avoids that identification of $\delta_{j} \mathrm{~s}$ in equation [1] suffers from endogeneity driven by unobservables that simultaneously affect mode and time of delivery. Indeed, the predicted probabilities at the first stage are an exogenous measure of the woman's ICS. In order to avoid that the coefficients of interest are solely identified though non linearity of the probability function, we rely on existing clinical literature to 
pin down some pre-determined risk factors $z_{i}$ that affect the timing of delivery only through their impact on individual's ICS, as assessed by clinicians. Therefore our net benefit function takes the following form:

$$
N B\left(\sigma_{i}\right)=\beta_{0}{ }^{\prime} x_{i}+\beta_{z}{ }^{\prime} z_{i}
$$

As far as the 1) patient inflow is homogenous in time and 2) no variation in the clinical assessment of admitted women at the onset of labor takes place throughout the day, the process determining the ICS will be invariant across time, allowing for the identification of the effect of ICS on the timing of delivery in equation [1]. In the empirical analysis that follows we provide evidence in support of condition 1) as a maintained assumption (at least across days of the week), while we take assumption 2) on a normative "as if" basis.

\section{EMPIRICAL ANALYSIS AND EVIDENCE}

\subsection{SAMPLE DESCRIPTIVE STATISTICS}

Table 2 presents the descriptive statistics for our dependent variables and key regressors. We briefly comment here on risk factors for a CS. The role and use of variables related to time of admission and delivery will be explained below. Our model for ICS controls for most of the risk factor considered in the literature. We decided to omit risk factors that might be reported as ex-post justifications for the performed CS, rather than being based on objective clinical assessment. For instance dystocia (failure of labor to progress), fetal distress (low fetal oxygen), and fetal disproportion (the fetus is too large to fit through the birth canal) have been considered as "somewhat subjective conditions ... sometimes be coded in order to justify a cesarean ex post" (see Grant (2005)). As we mentioned above, Spetz et al. (2001) provide evidence suggesting this possibility. We opted instead for the inclusion of objective clinical conditions like antepartum haemorrhage, placenta praevia, placental abruption, blood hypertension, anemia and impaired glucose tolerance which might be possibly revealed during labor. We also included factors like the newborn weight, the fetal gender and childbearing elsewhere coded as "pathological", that are all predetermined at admission despite possibly unknown to the clinicians. All these factors are assumed to affect both ICS and the timing of delivery as they are either revealed or evolving during labor. A further set 
of conditions affect the exact timing of delivery only via their impact on ICS: primiparity, previous CS, admission to hospital during childbearing, and gestational age at admission. These conditions are predetermined and known at admission.

\subsection{THE COMPOSITION OF ADMISSION INFLOWS}

It has been recently established that seasonal birth rhythms, i.e. month of conception and then after month of delivery, display systematic variations across sociodemographic groups. ${ }^{5}$ However, conditionally on month of admission, it is to be expected that nature distributes urgent deliveries and the onsets of labor uniformly across days of the week. We test here for the presence of compositional effects in the inflow of admission across months and days for our selected sample. We enlarge our perspective to any compositional effect due to socio-economic but also to clinical risk factors. We perform our testing by estimating multinomial logit regressions for month and day of admission.

Table 3 contains p-values of joint test of multinomial logit coefficients for month of admission, while Table 4 contains p-values of joint test of multinomial logit coefficients for day of admission. Our evidence clearly suggests that the inflow of admission is not homogeneous across months (Table 3 ) but it is homogeneous across days of the week (Table 4).

\section{TABLE 3 \& TABLE 4 here}

\subsection{INDICATION FOR CAESAREAN SECTION DELIVERY}

Table 5 reports our estimates for the probability of CS as a function of covariates, and time of admission dummies (year, month, and day).

\section{TABLE 5}

According to the joint test for the coefficient of month and day of admission being equal to zero, the probability of caesarean section delivery does not vary significantly across

${ }^{5}$ Buckles and Hungerman (2013) for instance found that winter births in the US are disproportionally due to teenagers and the unmarried (see also Bobak and Gjonca (2001)). 
months and days, conditional on covariates. This evidence supports our assumption of clinical assessment being homogenous in time. Most of the risk factors considered are significant and with the expected signs, while all SES variables prove to be uncorrelated with ICS. Moreover, the joint test on the significance of the coefficients of the set of clinical conditions that influence time of delivery only through ICS. and not directly. strongly rejects the null. Overall, this evidence supports our identification strategy, suggesting that the decision concerning the mode of delivery is mainly driven by clinical arguments and is stable in time.

\subsection{INDICATION FOR CAESAREAN SECTION DELIVERY AND TIMING OF DELIVERY}

The estimated model for CS provides us with predicted values for individuals' ICS, i.e. the predicted probability for CS. Under our assumptions, this measure is exogenous to the exact time of delivery. Thus, we plug it into the multinomial model for daily shift of delivery and obtain estimates that can be claimed to be causal.

As a first exercise we look for the effect of ICS on day of delivery and test whether the exact day of delivery is somehow manipulated in the short run (see Table 6) with respect to the nature's almost uniform distribution of births across days. This effect proves to be systematically null. The decisions on the day of delivery (taken by agent 2 in our set-up) are not influenced by ICS (as assessed by agent 1), our indicator for individual "systematic" riskiness for CS. In particular women with a high ICS do not have a lower probability to deliver on Sundays as suggested in the literature (Brown (1996), Spetz et al. (2001)). Therefore we might claim that in our sample "demand for leisure" or "risk aversion" on physician side does not affect the exact day of births.

\section{TABLE 6}

We turn next to our further research question and explore the pattern of within-day exact timing of delivery. Table 7 shows estimated marginal effects of ICS on shift of delivery. We find that the higher is ICS the higher is the probability to deliver in the afternoon or evening shift, while the lower is to deliver in late night and early morning. This last effect is very precisely estimated and it is the largest both in absolute and relative terms: a $10 \%$ increase in ICS leads to a $1.5 \%$ reduction in the likelihood to deliver early in the morning. 
Table 8 provides some simulations of the impact on shifts of delivery when ICS moves from median to higher/lower representative percentiles. Being assessed as a patient with high ICS (upper 90 percentile) leads to a 13\% to 19\% larger probability to deliver in the afternoon and to a $12 \%$ to $22 \%$ lower probability to deliver late at night or early morning comparing to a median ICS patient. Being assessed as a patient with low ICS (lower 10 percentile) leads to opposite but smaller effects on shift of delivery, i.e. lower probability to deliver in the afternoon and larger probability to deliver late at night or early morning comparing to a median ICS patient. This comparison suggests the presence of heterogeneous effects of ICS on time of delivery. Namely, the manipulation of the exact time of delivery is larger among the high risk individuals than among the low risk ones.

\section{TABLE 7}

\section{TABLE 8}

Our evidence implies the existence of a circadian rhythm of peaks and troughs in the likelihood of delivery for high ICS patients. The question arises of what comes first and causes the next. In other words do we have anticipation of high ICS deliveries in the afternoon followed by a reduced probability of delivery late at night \early in the morning next day or do we have the postponement of high ICS deliveries in the late night \early morning shift followed by a peak in the next afternoon? The first explanation would be consistent with both a "risk aversion" attitude to anticipate risky deliveries and a "demand for leisure" desire to escape from a large workload around the end of the $8 \mathrm{pm}$ shift. The second explanation is consistent with the former, less so with the latter.

To unravel this issue we estimate a simple probit model for the probability to deliver beyond the day of admission. We find that ICS positively affects this probability: the higher is ICS the lower is the probability to deliver in the same day of admission and therefore the higher is the probability to be postponed (see Table 9). Provided that the inflow of urgent deliveries and women at the onset of labor is homogeneous and given that $94 \%$ of them deliver within the day after admission we are tempted to conclude 
that short-run postponement of higher ICS cases late at night motivate by a "risk aversion" attitude can be the prevalent explanation.

\section{TABLE 9}

Finally (see Table 10) we look after heterogeneous effect of ICS on shift of delivery across day of admission. We don't find any significant difference in the short-run manipulation of deliveries across admissions occurring in weekdays vis-à-vis in weekends. The marginal effects of ICS look indistinguishable and exhibit the same pattern described above: postponement of high ICS deliveries at night/early morning followed by a peak in the following afternoon shift. This evidence reinforces our interpretation in favor of the "risk aversion" motivation behind the manipulation of exact timing of birth, as far as we basically don't observe any evidence of an higher propensity to anticipate CS in the afternoon shifts during weekends where the quest for leisure should be stronger.

\section{TABLE 10}

\section{Conclusions}

It has been repeatedly noticed that the observed timing of deliveries does not track the nature's almost uniform distribution of births. Physicians are alleged as responsible for this medical manipulation of the timing of deliveries. Several motivations have been suggested in the literature. The most relevant candidates are the financial motivation behind the Physician Demand Induction hypothesis, physicians' "aversion" to medical errors either benevolent or rational, as driven by medical malpractice, and the "demand for leisure" motivation.

We investigate this issue using birth certificates and hospital discharge records for a five years period (2007-2011) in one big public hospital of region Emilia-Romagna, Italy. Our setting negates the influence of financial incentives behind the Physician Demand Induction hypothesis while it allows for "risk aversion" to minimize medical errors and for "demand for leisure" motivation. In the Italian SSN financial incentives to induce demand for CS are poor to null provided that doctors operating in public hospitals are salaried and no component of their compensation is linked to team 
performance. Moreover in the hospital studied here a delivery protocol is in place to limit inappropriate use of CS delivery.

Upon careful selection of the caseload of women admitted for urgencies and at the onset of labor, we provide empirical support to patients' inflow being homogenous in time. We develop our empirical strategy on this sample having in mind a clinical set up where upon admission at the hospital the patient is assessed, based upon pre-existing conditions plus on-going ones, as being less or more likely in need for a CS. After the clinical assessment and conditioning on the individual indication for CS (ICS) a decision on timing of delivery is taken.

We don't find any evidence of manipulation of the exact day of birth. We notice instead a clear pattern of timing within day that is uniform across weekdays and weekends admission. We find that ICS does not affect the exact day of delivery but we measure a circadian rhythm of peaks and troughs in the likelihood of delivery for high ICS patients. This pattern is consistent with the postponement of high ICS deliveries in the late night $\backslash$ early morning shift. The lower probability of delivery at night among women with high ICS might reflect the effect of fatigue on the timing decisions, leading to defer "bad cases" to the fresh day staff. We argue that our evidence hardly supports the manipulation of timing of births as driven by medical staff's "demand for leisure". A "risk aversion" attitude to postpone risky deliveries from the early morning shifts seems to be the most appropriate explanation. 


\section{REFERENCES}

Bernis, C., \& Varea, C. (2012). Hour of Birth and Birth Assistance: From a Primate to a Medicalized Pattern? American Journal of Human Biology, 24, 14-21

Bobak, M, \& Gjonca, A. (2001). The seasonality of live birth is strongly influenced by socio-demographic factors. Human Reproduction, 16(7), 1512-1517.

Brown, HS. (1996). Physician demand for leisure: Implications for cesarean section rates. Journal of Health Economics, 15, 233-242.

Buckles, KS, \& Hungerman, DM. (2013). Season of birth and later outcomes: old questions, new answers. The Review of Economics and Statistics, 95(3), 711-724.

Burns, LR, Geller, SE, \& Wholey, DR. (1995). The effect of physician factors on the cesarean section decision. Medical Care, 33, 365-382.

Chandra, A, Cutler, D, \& Song, Z. (2012). Who Ordered That? The Economics of Treatment Choices in Medical Care. In Pauly, MV, Mcguire, TG \& Pita Barros, P. (eds.) Handbook of Health Economics, Volume 2, Elsevier, Amsterdam.

Cohen, A. (1983), Seasonal Daily Effect on the Number of Births in Israel. Journal of the Royal Statistical Society. Series C, 32(3), 228-35.

de Graaf, JP, Ravelli, ACJ, Visser, GHA, Hukkelhoven, C, Tong, WH, Bonsel, GJ, \& Steegers, EAP. (2010). Increased adverse perinatal outcome of hospital delivery at night. BJOG, $117,1098-1107$.

Dickert-Conlin, S, \& Chandra A. (1999). Taxes and the Timing of Births. Journal of Political Economy, 107 (1), 161-177.

Dodd, JM, Crowther, CA, Grivell, RM, \& Deussen, AR. (2014). Elective repeat caesarean section versus induction of labour for women with a previous caesarean birth. Cochrane Database of Systematic Reviews, $\mathrm{n}^{\circ} 12$.

Gans JS, \& Leigh A. (2008). What Explains the Fall in Weekend Births? Mimeo.

Gans, JS, \& Leigh, A. (2009). Born on the first of july: An (un)natural experiment in birth timing. Journal of Public Economics, 93(1-2), 246-263.

Gans, JS, \& Leigh, A. (2012). Bargaining over labour: Do patients have any power? Economic Record, 88(281), 182-194.

Gans, JS, Leigh, A, \& Varganova, E. (2007). Minding the shop: The case of obstetrics conferences. Social Science \& Medicine, 65(7), 1458-1465.

Gomes, UA, Silva AM, Bettiol, H, \& Barbieri M. (1999). Risk factors for the increasing caesarean section rate in Southeast Brazil: a comparison of two birth cohorts, 19781979 and 1994. International Journal of Epidemiology, 28, 687-694.

Gould, JB, Qin, C, \& Chavez, G. (2005). Time of Birth and the Risk of Neonatal Death. Obstetrics \& Gynecology, 106(2), 352-358. 
Grant, D. (2005). Explaning Source of Payments Differences in U.S. Cesarean Rates: Why Do Privately Insured Mothers Receive More Cesareans than Mothers Who Are Not Privately Insured? Health Care Management Science, 8, 5-17.

Hsu, K-H, Liao, P-J, \& Hwang, C-J. (2007). Factors affecting Taiwanese women's choice of cesarean section. Social Science \& Medicine, 66, 201-209.

Lefevre, M. (2014), Physician induced demand for C-sections: does the convenience incentive matter? HEDG working paper $\mathrm{n}^{\circ} 14 / 08$, University of York.

Lo, JC. (2003). Patients' attitudes vs. Physicians' determination: implications for cesarean sections. Social Science \& Medicine, 57, 91-96

Luo, ZC, \& Karlberg, J. (2001). Timing of birth and infant and early neonatal mortality in Sweden 1973-95: longitudinal birth register study. British Medical Journal, 323(December), 1-5.

Lydon-Rochelle MT, Cárdenas V, Nelson JC, Holt VL, Gardella C, \& Easterling TR. (2007). Induction of labor in the absence of standard medical indications: incidence and correlates. Medical Care, 45, 505-12.

Mossialos E., Allin S., Karras K. \& Davaki K. (2005). An investigation of Caesarean sections in three Greek hospitals. European Journal of Public Health, 15, (3), 288-295

Nassar AH, Adra AM, Chakhtoura N, Gómez-Marín 0, Beydoun S, 1998, "Severe preeclampsia remote from term: labor induction or elective cesarean delivery?" Am J Obstet Gynecol. 1998 Nov;179(5):1210-3.

Spetz, J, Smith MW, \& Ennis SF. (2001). Physician incentives and the timing of cesarean sections: evidence from California. Medical Care, 39, 536-550. 


\section{Tables and figures}

Figure 1: Deliveries across day hours.

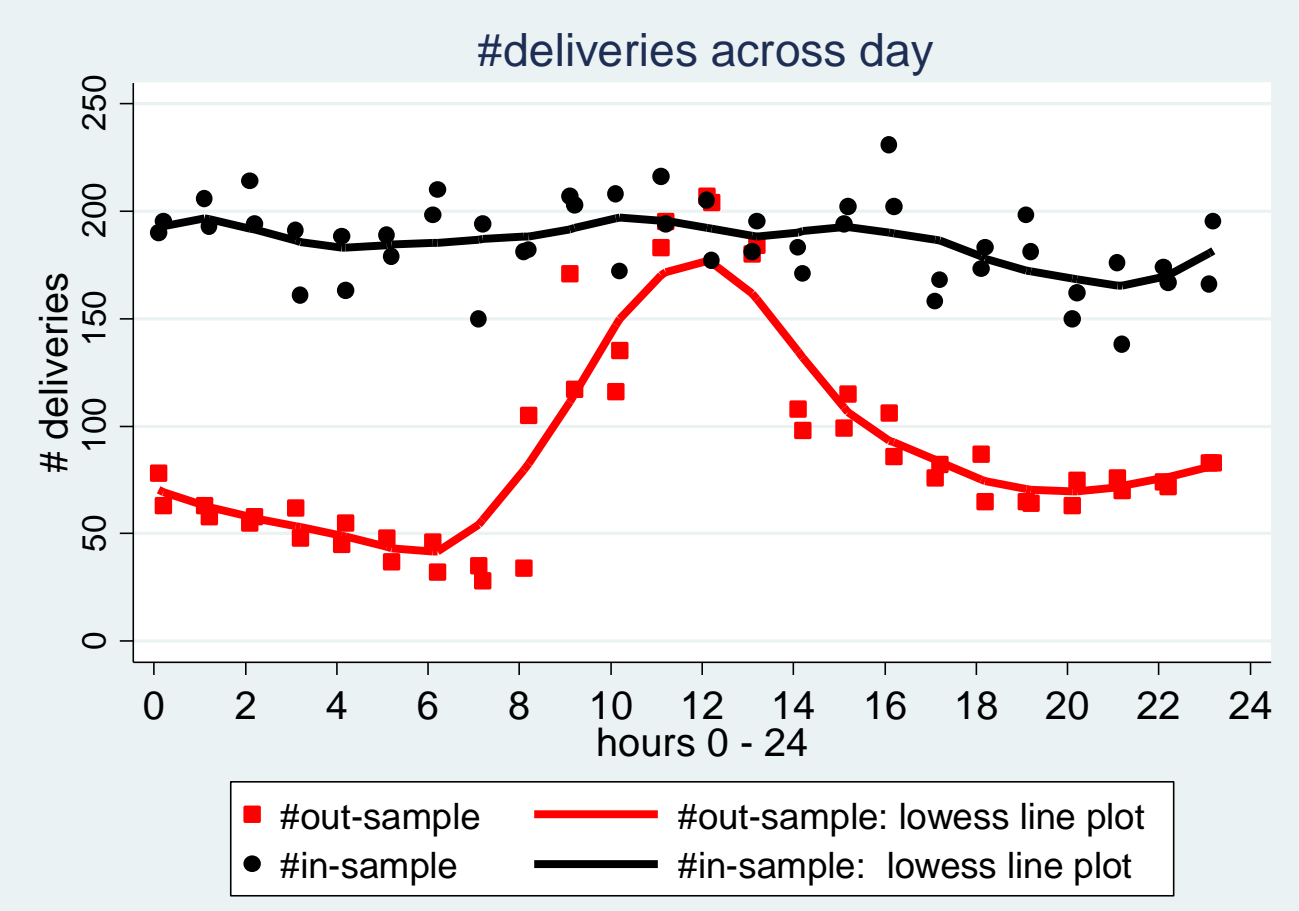

Figure 2: Cesarean section rates across day hours.

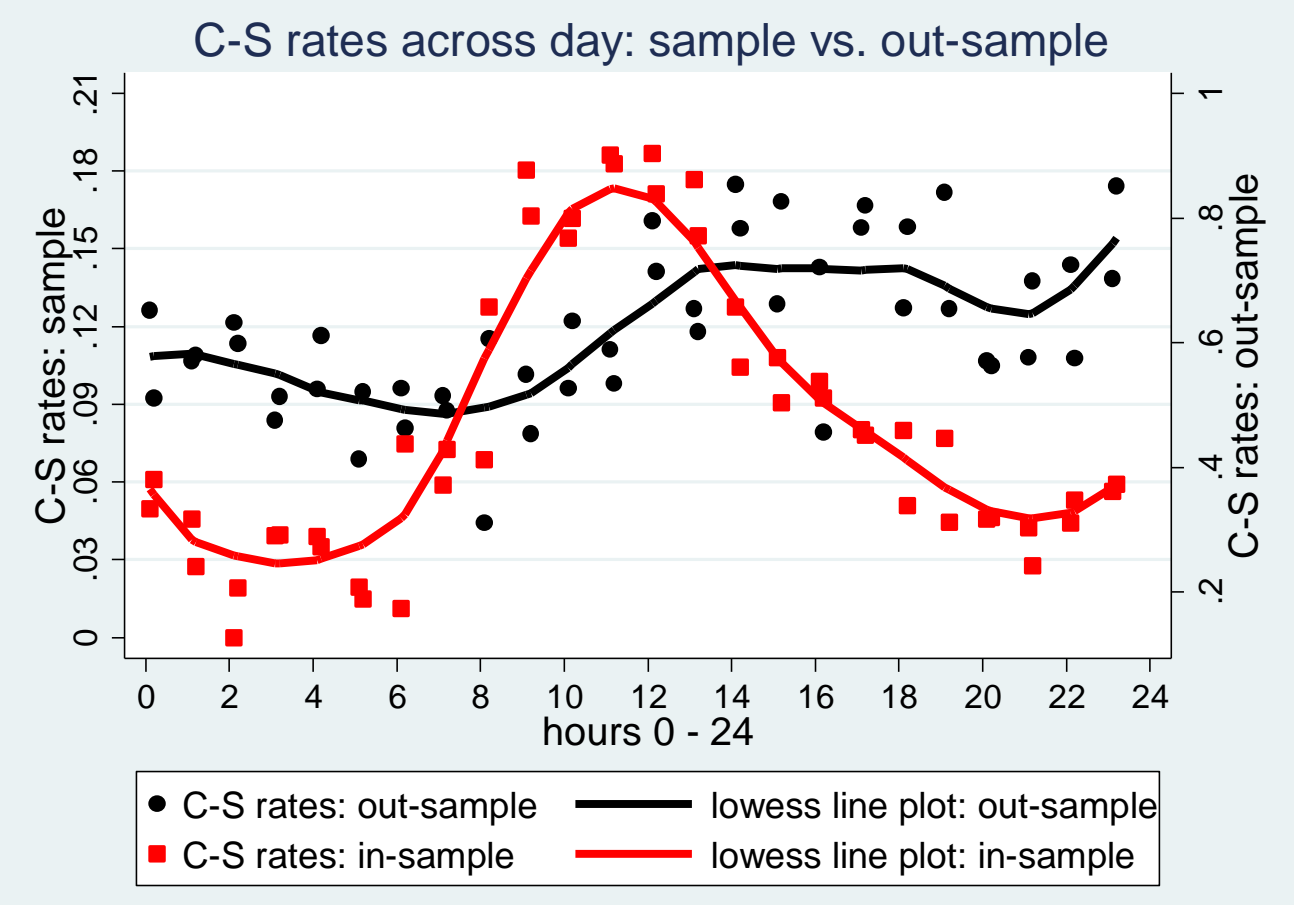


TABLE 1: Cesarean section rates and delivery rate by shift

\begin{tabular}{|c|c|c|c|c|c|c|}
\hline SHIFT & \# observations & CS rate & CS rate OR & $\begin{array}{c}\text { prob. to deliver by } \\
\text { shift }\end{array}$ & $\begin{array}{c}\text { prob. to deliver by } \\
\text { shift OR }\end{array}$ & $\begin{array}{c}\text { avg. \# deliveries in } \\
\text { the shift }\end{array}$ \\
\hline \multicolumn{7}{|l|}{ SELECTED SAMPLE } \\
\hline morning (8 am - 11 am) & 1153 & 0.093 & 0.79 & 0.129 & 1.03 & 0.632 \\
\hline late morning (11 am - 2 pm) & 1168 & 0.126 & 1.07 & 0.131 & 1.05 & 0.640 \\
\hline afternoon (2 pm - 5 pm) & 1183 & 0.141 & 1.20 & 0.133 & 1.06 & 0.648 \\
\hline late afternoon (5 pm - 8 pm) & 1061 & 0.152 & 1.29 & 0.119 & 0.95 & 0.581 \\
\hline evening (8 pm - 11 pm) & 967 & 0.118 & 1.00 & 0.109 & 0.87 & 0.530 \\
\hline night (11 pm - 2 am) & 1145 & 0.124 & 1.05 & 0.129 & 1.02 & 0.627 \\
\hline late night (2 am - 5 am) & 1111 & 0.104 & 0.88 & 0.125 & 0.99 & 0.609 \\
\hline early morning ( 5 am - 8 am) & 1120 & 0.087 & 0.73 & 0.126 & 1.00 & 0.614 \\
\hline ALL DAY & 8908 & 0.118 & 1.00 & 0.125 & 1.00 & 0.612 \\
\hline \multicolumn{7}{|l|}{ EXCLUDED OBSERVATIONS } \\
\hline morning (8 am - 11 am) & 678 & 0.773 & 1.35 & 0.158 & 1.01 & 0.372 \\
\hline late morning (11 am - 2 pm) & 1153 & 0.861 & 1.50 & 0.269 & 1.72 & 0.632 \\
\hline afternoon (2 pm - 5 pm) & 612 & 0.559 & 0.97 & 0.143 & 0.91 & 0.335 \\
\hline late afternoon (5 pm - 8 pm) & 439 & 0.417 & 0.73 & 0.102 & 0.65 & 0.241 \\
\hline evening (8 pm - 11 pm) & 430 & 0.307 & 0.53 & 0.100 & 0.64 & 0.236 \\
\hline night (11 pm - 2 am) & 428 & 0.339 & 0.59 & 0.100 & 0.64 & 0.235 \\
\hline late night (2 am - 5 am) & 323 & 0.245 & 0.43 & 0.075 & 0.48 & 0.177 \\
\hline early morning ( 5 am - 8 am) & 226 & 0.283 & 0.49 & 0.053 & 0.34 & 0.124 \\
\hline ALL DAY & 4289 & 0.574 & 1.00 & 0.157 & 1.00 & 0.368 \\
\hline
\end{tabular}

Note: Excluded observations are deliveries explicitly classified as "elective" CS and also those occurring upon induced labor not for emergencies arising at term. 
TABLE 2: Descriptive statistics.

\begin{tabular}{|c|c|c|}
\hline VARIABLE & MEAN & $\begin{array}{l}\text { STD. } \\
\text { DEV. }\end{array}$ \\
\hline CESAREAN SECTION delivery & 0.118 & 0.323 \\
\hline \multicolumn{3}{|l|}{ SHIFT } \\
\hline morning (8 am - 11 am) & 0.129 & 0.336 \\
\hline late morning (11 am - 2 pm) & 0.131 & 0.338 \\
\hline afternoon ( 2 pm - 5 pm) & 0.133 & 0.339 \\
\hline late afternoon ( 5 pm - 8 pm) & 0.119 & 0.324 \\
\hline evening ( 8 pm - 11 pm) & 0.109 & 0.311 \\
\hline night (11 pm - 2 am) & 0.129 & 0.335 \\
\hline late night ( 2 am - 5 am) & 0.125 & 0.330 \\
\hline early morning ( 5 am - 8 am) & 0.126 & 0.332 \\
\hline \multicolumn{3}{|l|}{ Year of admission } \\
\hline 2007 & 0.195 & 0.396 \\
\hline 2008 & 0.191 & 0.393 \\
\hline 2009 & 0.187 & 0.390 \\
\hline 2010 & 0.195 & 0.396 \\
\hline 2011 & 0.232 & 0.422 \\
\hline \multicolumn{3}{|l|}{ Month of admission } \\
\hline January & 0.080 & 0.272 \\
\hline February & 0.075 & 0.264 \\
\hline March & 0.088 & 0.283 \\
\hline April & 0.071 & 0.257 \\
\hline May & 0.082 & 0.274 \\
\hline June & 0.083 & 0.276 \\
\hline July & 0.093 & 0.290 \\
\hline August & 0.081 & 0.273 \\
\hline September & 0.093 & 0.291 \\
\hline October & 0.089 & 0.284 \\
\hline November & 0.084 & 0.277 \\
\hline December & 0.081 & 0.273 \\
\hline \multicolumn{3}{|l|}{ Day of admission } \\
\hline Monday & 0.147 & 0.354 \\
\hline Tuesday & 0.146 & 0.353 \\
\hline Wednesday & 0.137 & 0.344 \\
\hline Thursday & 0.140 & 0.347 \\
\hline Friday & 0.145 & 0.352 \\
\hline Saturday & 0.142 & 0.349 \\
\hline Sunday & 0.143 & 0.350 \\
\hline \multicolumn{3}{|l|}{ PRE-DETERMINED RISK FACTORS* } \\
\hline Primiparity & 0.555 & 0.497 \\
\hline Delivered at least twice in the past & 0.095 & 0.294 \\
\hline Previous c-section & 0.046 & 0.210 \\
\hline Admitted to hospital during childbearing & 0.029 & 0.168 \\
\hline Delivery before 35 weeks & 0.028 & 0.166 \\
\hline Delivery after 41 weeks & 0.169 & 0.375 \\
\hline \multicolumn{3}{|l|}{ RISK FACTORS FOR CS } \\
\hline Fetal gender is male & 0.517 & 0.500 \\
\hline Weight of the newborn below $2.5 \mathrm{~kg}$ & 0.044 & 0.205 \\
\hline Weight of the newborn above $4.0 \mathrm{~kg}$ & 0.071 & 0.257 \\
\hline Childbearing considered "pathological" & 0.073 & 0.260 \\
\hline Antepartum haemorrhage, placenta praevia, placental abruption & 0.009 & 0.093 \\
\hline Suffering from blood hypertension & 0.021 & 0.144 \\
\hline Suffering from anemia & 0.063 & 0.244 \\
\hline
\end{tabular}




\begin{tabular}{lcc} 
Impaired glucose tolerance & 0.015 & 0.122 \\
SES VARIABLES & & \\
Age & 32.010 & 5.381 \\
Age squared & 1053.6 & 336.4 \\
Holding a middle school diploma & 0.215 & 0.411 \\
Holding a high school diploma & 0.449 & 0.497 \\
Holding a university diploma & 0.005 & 0.072 \\
Holding a BA/BS or more & 0.310 & 0.463 \\
Married & 0.650 & 0.477 \\
Employed & 0.749 & 0.434 \\
& & \\
\# OBS & 8908 & \\
\hline
\end{tabular}

Note: *Pre-determined risk factors affecting SHIFT only via ICS. 
TABLE 3: Multinomial logit model for month of admission: p-values of joint test of coefficients of covariates (by subgroups and all)

\begin{tabular}{lcccc}
\hline Month of admission & $\begin{array}{c}\text { Pre-determined } \\
\text { risk factors }\end{array}$ & Risk factors & SES & ALL \\
\cline { 2 - 5 } February & 0.165 & 0.000 & 0.866 & 0.000 \\
March & 0.002 & 0.426 & 0.107 & 0.029 \\
April & 0.117 & 0.062 & 0.006 & 0.001 \\
May & 0.026 & 0.291 & 0.121 & 0.030 \\
June & 0.080 & 0.551 & 0.093 & 0.036 \\
July & 0.020 & 0.034 & 0.300 & 0.001 \\
August & 0.005 & 0.014 & 0.020 & 0.000 \\
September & 0.035 & 0.198 & 0.013 & 0.005 \\
October & 0.003 & 0.073 & 0.066 & 0.000 \\
November & 0.005 & 0.273 & 0.039 & 0.007 \\
December & 0.008 & 0.315 & 0.009 & 0.000 \\
ALL MONTHS & & & & \\
\hline
\end{tabular}

TABLE 4: Multinomial logit model for day of admission: p-values of joint test of coefficients of covariates (by subgroups and all)

\begin{tabular}{lcccc}
\hline Day of admission & $\begin{array}{c}\text { Pre-determined } \\
\text { risk factors }\end{array}$ & Risk factors & SES & ALL \\
\cline { 2 - 5 } Tuesday & 0.974 & 0.245 & 0.950 & 0.905 \\
Wednesday & 0.724 & 0.315 & 0.765 & 0.644 \\
Thursday & 0.404 & 0.624 & 0.506 & 0.530 \\
Friday & 0.984 & 0.475 & 0.732 & 0.832 \\
Saturday & 0.885 & 0.455 & 0.753 & 0.717 \\
Sunday & 0.578 & 0.040 & 0.299 & 0.023 \\
& & & & \\
ALL DAYS & 0.676 & 0.299 & 0.499 & 0.237 \\
\hline
\end{tabular}


TABLE 5: Indication to Caesarean Section probit model: probability of CS as a function of covariates and admission (year, month and day).

\begin{tabular}{|c|c|c|}
\hline & COEFFICIENT & $\begin{array}{l}\text { STANDARD } \\
\text { ERROR }\end{array}$ \\
\hline \multicolumn{3}{|l|}{ Year of admission (base $=2007$ ) } \\
\hline 2008 & $0.122 * *$ & $(0.062)$ \\
\hline 2009 & 0.051 & $(0.062)$ \\
\hline 2010 & -0.081 & $(0.064)$ \\
\hline 2011 & $-0.124 * *$ & $(0.062)$ \\
\hline \multicolumn{3}{|l|}{ Month of admission (base $=$ January) } \\
\hline February & -0.105 & $(0.100)$ \\
\hline March & -0.088 & $(0.097)$ \\
\hline April & 0.034 & $(0.100)$ \\
\hline May & 0.023 & $(0.098)$ \\
\hline June & 0.029 & $(0.097)$ \\
\hline July & -0.098 & $(0.097)$ \\
\hline August & -0.050 & (0.099) \\
\hline September & -0.034 & $(0.095)$ \\
\hline October & -0.012 & $(0.095)$ \\
\hline November & 0.000 & $(0.097)$ \\
\hline December & -0.117 & (0.099) \\
\hline \multicolumn{3}{|l|}{ Day of admission (base = Monday) } \\
\hline Tuesday & -0.008 & $(0.072)$ \\
\hline Wednesday & -0.075 & $(0.072)$ \\
\hline Thursday & 0.012 & $(0.073)$ \\
\hline Friday & -0.066 & $(0.073)$ \\
\hline Saturday & 0.008 & $(0.071)$ \\
\hline Sunday & -0.122 & $(0.075)$ \\
\hline \multicolumn{3}{|l|}{ PRE-DETERMINED RISK FACTORS } \\
\hline Primiparity & $0.775^{* * *}$ & $(0.054)$ \\
\hline Delivered at least twice in the past & -0.107 & $(0.090)$ \\
\hline Previous cesarean section & $1.901 * * *$ & $(0.078)$ \\
\hline Admitted to hospital during childbearing & $0.259 * *$ & (0.109) \\
\hline Admitted pre-term (before 35 weeks of gestation) & 0.169 & $(0.138)$ \\
\hline Admitted after-term (after 41 weeks of gestation) & $0.248 * * *$ & $(0.050)$ \\
\hline \multicolumn{3}{|l|}{ RISK FACTORS } \\
\hline Fetal gender is male & $0.170 * * *$ & $(0.040)$ \\
\hline Weight of the newborn below $2.5 \mathrm{~kg}$ & $0.642 * * *$ & $(0.103)$ \\
\hline Weight of the newborn above $4.0 \mathrm{~kg}$ & 0.115 & $(0.080)$ \\
\hline Childbearing considered "pathological" & $0.266 * * *$ & $(0.077)$ \\
\hline Antepartum haemorrhage, placenta praevia, placental abruption & $1.931^{* * *}$ & $(0.212)$ \\
\hline Suffering from blood hypertension & $1.240 * * *$ & $(0.112)$ \\
\hline Suffering from anemia & $-0.566 * * *$ & $(0.099)$ \\
\hline Impaired glucose tolerance & 0.215 & $(0.155)$ \\
\hline \multicolumn{3}{|l|}{ SES VARIABLES } \\
\hline Age & 0.001 & $(0.034)$ \\
\hline Age squared & 0.001 & $(0.001)$ \\
\hline Holding a middle school diploma & -0.097 & $(0.151)$ \\
\hline Holding a high school diploma & -0.123 & $(0.150)$ \\
\hline Holding a university diploma & -0.060 & $(0.285)$ \\
\hline Holding a BA/BS or more & $-0.264^{*}$ & $(0.152)$ \\
\hline
\end{tabular}


Day of admission $=0$

$\mathrm{Chi}^{2}$ joint test

p-value

Month of admission $=0$

5.706

0.457

Pre-determined risk factors $=0$

7.071

0.793

637.520

0.000

Pseudo $\mathrm{R}^{2}$

0.233

N. of observations

8908

Robust standard errors in parentheses.

$* * *, * *, *$ denote significance levels at $1 \%, 5 \%, 10 \%$.

TABLE 6: Estimated marginal effects of indication to cesarean section (ICS) on day of delivery

\begin{tabular}{lcc}
\hline & Marginal effect & Standard Error \\
\cline { 2 - 3 } MONDAY & 0.017 & $(0.033)$ \\
TUESDAY & 0.004 & $(0.028)$ \\
WEDNESDAY & -0.007 & $(0.029)$ \\
THURSDAY & 0.026 & $(0.030)$ \\
FRIDAY & -0.002 & $(0.031)$ \\
SATURDAY & -0.013 & $(0.029)$ \\
SUNDAY & -0.026 & $(0.033)$ \\
& & \\
ICS =0 & Chi $^{2}$ joint test & p-value \\
& 1.924 & 0.927 \\
WEEKDAY & & \\
WEEKEND & 0.035 & $(0.038)$ \\
& -0.035 & $(0.038)$ \\
ICS $=0$ & & $p$-value \\
\hline
\end{tabular}

Bootstrapped standard errors in parentheses (500 replications). 
TABLE 7: Estimated effects of indication to cesarean section (ICS) on shift of delivery

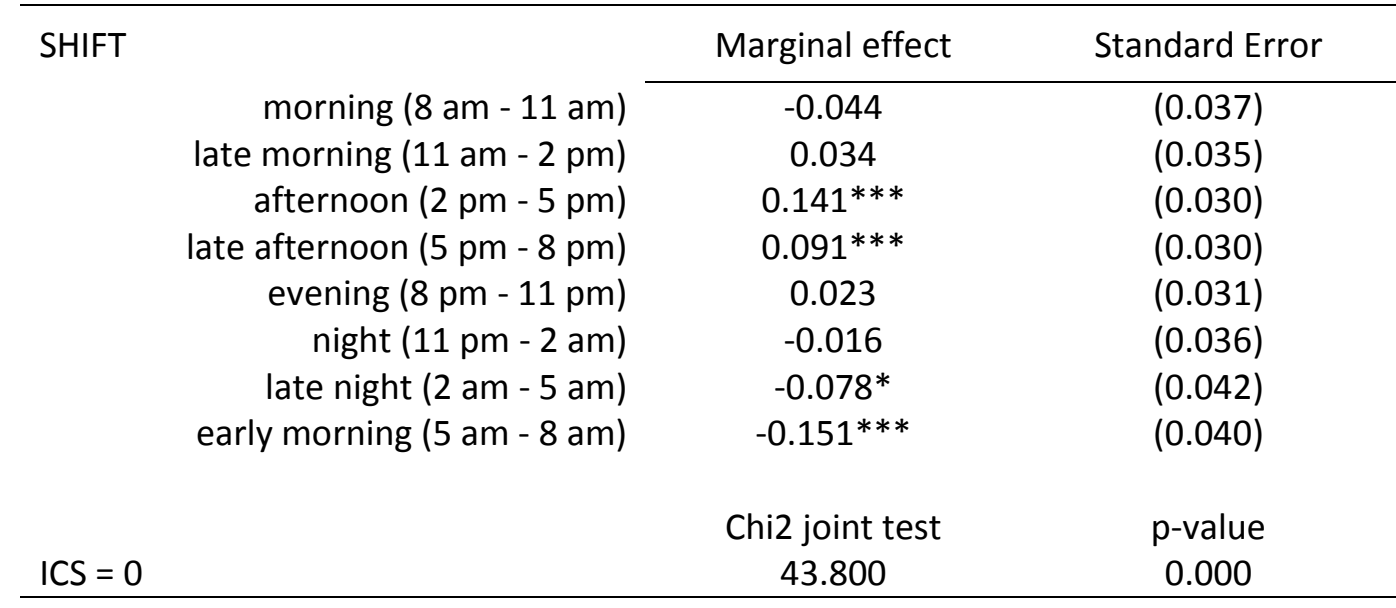

Bootstrapped standard errors in parentheses (500 replications).

$* * *, * *, *$ denote significance levels at $1 \%, 5 \%, 10 \%$.

TABLE 8: Change in the predicted probability of delivery by shift as for changes in the quantiles of indication to cesarean section (ICS)

\begin{tabular}{|c|c|c|c|c|c|}
\hline \multirow[t]{2}{*}{ SHIFT } & \multirow{2}{*}{$\begin{array}{l}\text { Prediction } \\
\text { at median }\end{array}$} & \multicolumn{4}{|c|}{$\begin{array}{l}\text { Change in the predicted probability of delivery by shift as } \\
\text { propensity to CS goes from median to: }\end{array}$} \\
\hline & & $10^{\text {th }}$ pctile & $25^{\text {th }}$ pctile & $75^{\text {th }}$ pctile & $90^{\text {th }}$ pctile \\
\hline morning (8 am - 11 am) & 0.131 & $1.7 \%$ & $1.3 \%$ & $-1.9 \%$ & $-6.5 \%$ \\
\hline late morning (11 am - $2 \mathrm{pm}$ ) & 0.130 & $-1.7 \%$ & $-1.2 \%$ & $1.5 \%$ & $4.3 \%$ \\
\hline afternoon (2pm- $5 \mathrm{pm})$ & 0.126 & $-6.2 \%$ & $-4.7 \%$ & $6.1 \%$ & $18.7 \%$ \\
\hline late afternoon (5 pm - $8 \mathrm{pm})$ & 0.115 & $-4.4 \%$ & $-3.4 \%$ & $4.4 \%$ & $13.2 \%$ \\
\hline evening ( $8 \mathrm{pm}-11 \mathrm{pm}$ ) & 0.107 & $-1.4 \%$ & $-1.1 \%$ & $1.3 \%$ & $3.5 \%$ \\
\hline night (11 pm - 2 am) & 0.130 & $0.5 \%$ & $0.4 \%$ & $-0.7 \%$ & $-2.7 \%$ \\
\hline late night (2 am - 5 am) & 0.128 & $3.3 \%$ & $2.5 \%$ & $-3.5 \%$ & $-11.7 \%$ \\
\hline early morning ( 5 am - 8 am) & 0.132 & $6.5 \%$ & $5.0 \%$ & $-6.8 \%$ & $-22.0 \%$ \\
\hline
\end{tabular}


TABLE 9: Estimated effects of indication to cesarean section (ICS) on the probability of delivering beyond day of admission

\begin{tabular}{rcc}
\hline ADMISSION on: & Marginal effect & Standard Error \\
\cline { 3 - 3 } Any day & $0.496^{* * *}$ & $(0.060)$ \\
Weekday & $0.490^{* * *}$ & \\
Weekend (SAT or SUN) & $0.515^{* * *}$ & $(0.062)$ \\
& & $(0.085)$ \\
Weekday & $0.492^{* * *}$ & \\
Weekend (FRI or SUN) & $0.482^{* * *}$ & $(0.065)$ \\
Weekend (SUN) & $0.579^{* * *}$ & $(0.077)$ \\
\hline
\end{tabular}

Bootstrapped standard errors in parentheses (500 replications).

$* * *, * *, *$ denote significance levels at $1 \%, 5 \%, 10 \%$.

TABLE 10: Marginal effects of indication to cesarean section (ICS) on shift of delivery by day of admission: weekday vs. weekend

\begin{tabular}{|c|c|c|c|c|c|}
\hline \multirow[t]{2}{*}{ SHIFT } & \multicolumn{2}{|c|}{ Admission in a weekday } & \multicolumn{2}{|c|}{ Admission in a weekend } & \multirow[t]{2}{*}{$p$-value } \\
\hline & Marginal effect & Standard Error & Marginal effect & Standard Error & \\
\hline morning (8 am - 11 am) & -0.048 & $(0.040)$ & -0.029 & $(0.034)$ & 0.748 \\
\hline late morning (11 am - 2 pm) & 0.061 & $(0.059)$ & -0.052 & $(0.044)$ & 0.075 \\
\hline afternoon (2 pm - 5 pm) & $0.151 * * *$ & $(0.037)$ & $0.114^{* * *}$ & $(0.038)$ & 0.486 \\
\hline late afternoon (5 pm - 8 pm) & 0.080 & $(0.062)$ & $0.122 * *$ & $(0.056)$ & 0.402 \\
\hline evening (8 pm - 11 pm) & 0.008 & $(0.032)$ & 0.068 & $(0.044)$ & 0.186 \\
\hline night (11 pm - 2 am) & -0.017 & $(0.051)$ & -0.013 & $(0.069)$ & 0.944 \\
\hline late night (2 am - 5 am) & $-0.070 * *$ & $(0.034)$ & $-0.103^{* *}$ & $(0.042)$ & 0.634 \\
\hline \multirow[t]{2}{*}{ early morning ( 5 am - 8 am) } & $-0.165^{* * *}$ & $(0.045)$ & $-0.108^{*}$ & $(0.065)$ & 0.370 \\
\hline & $\mathrm{Chi}^{2}$ joint test & $p$-value & & & \\
\hline $\mathrm{ICS}=0$ & 51.109 & 0.000 & & & \\
\hline$I C S(W D)=I C S(W E)$ & 6.886 & 0.441 & & & \\
\hline
\end{tabular}

Bootstrapped standard errors in parentheses (500 replications).

$* * *, * *, *$ denote significance levels at $1 \%, 5 \%, 10 \%$. 


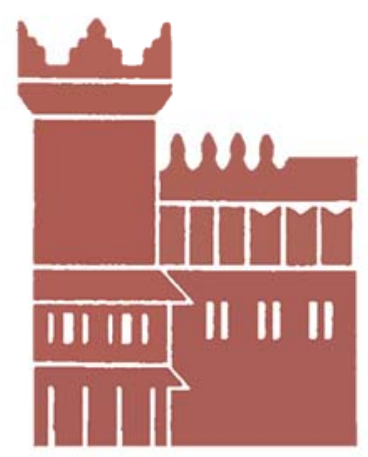

Alma Mater Studiorum - Università di Bologna DEPARTMENT OF ECONOMICS

Strada Maggiore 45

40125 Bologna - Italy

Tel. +39051 2092604

Fax +390512092664

http://www.dse.unibo.it 